\title{
General theory of three-dimensional radiance measurements with optical microprobes
}

\author{
N. Fukshansky-Kazarinova, L. Fukshansky, M. Kühl, and B. B. Jørgensen
}

\begin{abstract}
Measurements of the radiance distribution and fluence rate within turbid samples with fiber-optic radiance microprobes contain a large variable instrumental error caused by the nonuniform directional sensitivity of the microprobes. A general theory of three-dimensional radiance measurements is presented that provides correction for this error by using the independently obtained function of the angular sensitivity of the microprobes. (C) 1997 Optical Society of America

Key words: Optics of living tissue; radiance measurements; fiber-optic microprobe; three-dimensional picture of a light field in turbid media.
\end{abstract}

\section{Introduction}

Studies of light-controlled processes in photomedicine, photobiology, and ecology require a detailed knowledge of the light microenvironment within absorbing turbid media such as living tissue and sediments. Direct three-dimensional measurements of radiation fields in such samples with optical fiber microprobes have been increasingly employed over the past several years. ${ }^{1-9}$

The most universal type of optical fiber probe is the radiance microprobe, which has a tip diameter as small as $10 \mu \mathrm{m}$ and a directional sensitivity mainly concentrated around the axis of the probe within a solid angle as small as $10^{\circ}$. In contrast to other types that sense the entire spherical or hemispherical flux,$^{8}$ it is the only probe that provides the angular distribution of radiance. A radiance distribution at a given depth is found on the basis of successive measurements with a radiance microprobe advanced to this depth in different zenithal directions, $\theta$, relative to the light source as shown in Fig. 1. The unit sphere containing all directions (Fig. 1) is subdivided into $K$ spherical bands, and within each band $i$ one measurement under the angle $\theta_{i}$ is carried out. This

L. Fukshansky is with the Department of Biology, University of Freiburg, Schanzlestrausse 1, D-79104 Freiburg, Germany. The other authors are with the Max Planck Institute of Marine Microbiology, Microsensor Research Group, Celsiusstrausse 1, D-28359 Bremen, Germany.

Received 24 April 1996; revised manuscript received 5 December 1996.

0003-6935/97/256520-09\$10.00/0

(C) 1997 Optical Society of America measurement is representative for the radiance in any direction within the band number $i$, i.e., the radiance within each band is assumed to be constant. We designate this radiance value as $L_{i}$. To the accuracy of this discretization the solid radiance distribution is given by the sequence of measured values $L_{i}: L(\theta)=\left\{L_{1}, L_{2}, \ldots, L_{k}\right\}$.

The fluence rate $I(P)$ in a point $\mathrm{P}$ (see Fig. 1) is then obtained as a sum of $L_{i}$ weighted with the fractional areas of the corresponding spherical bands, $w_{i}$ :

$$
I(P)=2 \pi \int_{\theta} L(\theta) d \theta \approx 4 \pi \sum_{i=1}^{K} L_{i} w_{i}
$$

The weighting factors $w_{i}$ are easily derived from the geometrical considerations as

$$
\begin{aligned}
w_{i} & =\left(\cos \bar{\theta}_{i-1}-\cos \bar{\theta}_{i}\right) / 2, \\
\bar{\theta}_{0} & =0, \bar{\theta}_{K}=\pi, \quad \sum_{i} w_{i}=1,
\end{aligned}
$$

where $\bar{\theta}_{i-1}, \bar{\theta}_{i}$ are the zenithal angles for the boundary circles on the unit sphere delimiting the band $i$ (see Fig. 1). Importantly, a measurement in this scheme in principle yields not the radiance $L_{i}$ but the radiant flux $L_{i} S$, where $S$ is the reference area of the microprobe on the unit sphere ( $S$ is shown as a bright circle in Fig. 1). Thus the reading of a measurement should be divided by $S$ to obtain $L_{i}$.

However, as shown elsewhere, ${ }^{10}$ the described measurements produce erroneous values of $L_{i}$. This intrinsic instrumental error results from the nonuniform angular sensitivity of the microprobe.

Radiance microprobes (for technical details see Refs. 2, 4, and 6) have well-defined light-collecting 


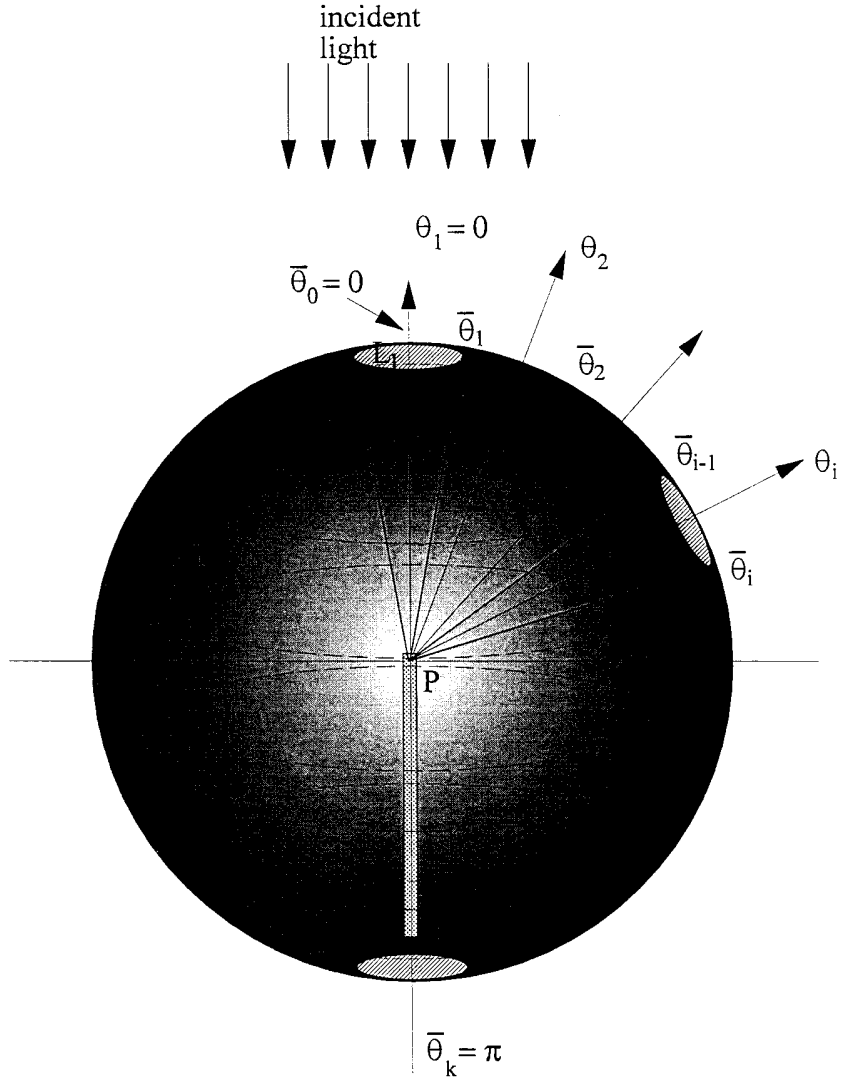

Fig. 1. Spatial design of three-dimensional radiance measurements. To perform the measurement number $i$, the microprobe advanced to the point $\mathrm{P}$ is oriented in the direction $\theta_{i}(i=1,2, \ldots$, $K)$. Each measurement is representative of the radiance within the corresponding spherical band of the unit sphere circumscribing the point $\mathrm{P}$. The radiance value $L_{i}$ is assumed to be constant within the band number $i$. $\bar{\theta}_{i-1}, \bar{\theta}_{i}$ are the zenithal angles of the circles delimiting the band $i$.

properties. The directional sensitivity of a radiance microprobe is specified by a numerical aperture $n_{0}$ $\sin \left(\theta_{a}\right)$, where $n_{0}$ is the refraction index of the medium and $\theta_{a}$ is the acceptance half-angle of the optical fiber. ${ }^{11}$ The meaning of $\theta_{a}$ is clear from Fig. 2, which shows examples of the angular sensitivity distribution of a probe $h$ as a function of the deviation $v$ from the optical axis of the probe. The sensitivity $h(v)$, being maximal along the axis of the probe, decreases monotonously with $v$. The angle $\theta_{a}$ specifies such deviation $v$ for which $h(v)=0.5 h_{\text {max }}$. The bellshaped distribution $h(v)$ is directly measurable. It can be approximated by a slightly modified Gaussian formula: ${ }^{10}$

$$
h(v)=\cos (v) \exp \left[-m \sin ^{2}(v)\right]
$$

where the fitting coefficient $m$ adjusts the function to the individual curve of the microprobe under consideration. Parameters $m$ and $\theta_{a}$ are related as

$$
m=\ln \left(2 \cos \theta_{a}\right) / \sin ^{2} \theta_{a} .
$$

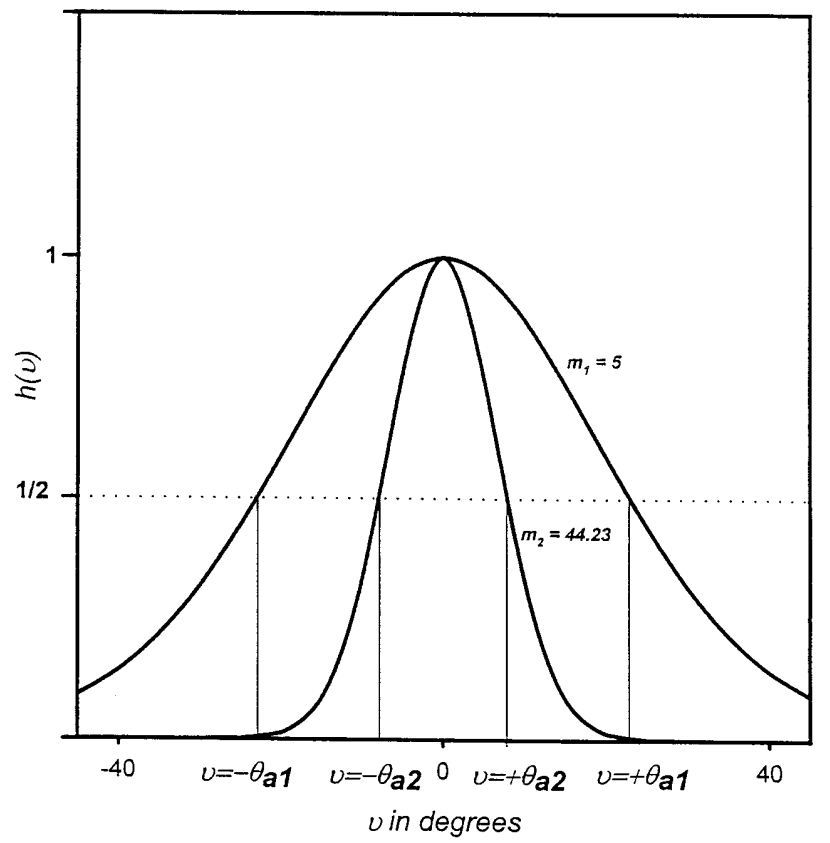

Fig. 2. Angular sensitivity distribution of a microprobe: $v$ is the internal angular coordinate; $v=0$ concides with the axis of the probe; $h(v)$ is the relative sensitivity [see Eq. (2)], $h(v)$ is maximal for $v=0$ while light deviating from the axis is perceived with lower sensitivity. The acceptance half-angle $\pm \theta_{a}$ specifies the $v$ values for which $h(v)=0.5 h_{\max }$. Function $h(v)$ is specified for a concrete probe by fixing either parameter $m$ or angle $\theta_{a}$. The two curves represent a flat $\left(m_{1}=5 ; \theta_{a 1}=21.2^{\circ}\right)$ and a strongly peaked $\left(m_{2}=\right.$ 44.23; $\left.\theta_{a 2}=7.2^{\circ}\right)$ sensitivity distribution.

Figure 2 shows two functions $h(v)$, with $m=44.23\left(\theta_{a}\right.$ $\left.=7.2^{\circ}\right)$ and $m=5\left(\theta_{a}=21.2^{\circ}\right)$. Both Eq. (2) and Fig. 2 present the normalized function $h(v)$, i.e., $h_{\max }=1$.

If a probe with the sensitivity distribution $h(v)$ such as that in Fig. 2 is used to measure $L_{i}$ in the scheme shown in Fig. 1, its optical axis has the orientation $\theta_{i}$, thus providing $v=0$ for $\theta=\theta_{i}$. Only the radiance directed exactly along $\theta_{i}$ is perceived with maximal sensitivity. The sensitivity for those radiances $L_{i}$ that belong to the band $i$ (e.g., with $\bar{\theta}_{i-1}<\theta<\bar{\theta}_{i}$ ) but deviate from the direction $\theta_{i}$ is decreasing with increasing deviation from $\theta_{i}$, as prescribed by the function $h(v)$. Furthermore, the probe also senses radiances from the neighboring bands. Thus any single measurement produces not the average radiance within the targeted band but a linear combination of different radiances weighted by the function $h(v)$. This measured quantity, which we designate as $M_{i}$, deviates from $L_{i}$.

In a recent paper ${ }^{10}$ we reported on the first step toward the analysis of the instrumental error and stated the problem of theoretical correction of the measurements. The idea of this approach was to process the measured data by using additional information contained in the function $h(v)$ that is measured independently. We developed this theory for the special case of equidistant measurements and accounted for the distorting contributions of only two immediate neighbors (i.e., bands $i-1$ and $i+1$ ). 
In this paper the general theory for arbitrarily spaced measurements is presented. In Subsection 2.A the problem is formulated in mathematical terms and the basic system of linear equations connecting $L_{i}$ and $M_{i}$ is derived. The coefficients of the equations are surface integrals of $h(v)$, with the complicated domains of integration arising from the geometry of measurements. Subsection 2.B contains an elucidation of these domains that is the prerequisite for the solution of the integrals given in Subsection 2.C. In Subsection 2.D we consider an application of the theory to our measurements in a costal sediment with diatoms. This treatment yields the instrumental error for the measured radiance distribution and fluence rate. It also shows that the divergent measured values obtained on the same sample with different probes converge very well after they were treated by the correcting procedure.

\section{Results}

\section{A. Mathematical Formulation of the Problem}

Let us consider radiation in an arbitrary point $\mathrm{P}$ within a turbid sample illuminated from above as shown in Fig. 1. Introducing spherical coordinates $\theta$ (zenith angle) and $\Psi$ (azimuth angle) associated with the unit sphere circumscribing $P$, we assume that at any point $(\theta, \Psi)$ the radiance depends on $\theta$ but not on $\Psi$. As described in Section $1, K$ measurements with a microprobe having the directional sensitivity distribution $h(v)$ are performed in the point $\mathrm{P}$ in the directions $\theta_{i}$ :

$$
0 \leq \theta_{1}<\theta_{2} \cdots<\theta_{K} \leq \pi .
$$

The results of these measurements are designated $M_{i}(i=1,2, \ldots, K)$. The unit sphere is subdivided into $K$ spherical bands (see Section 1), and radiance within each band is assumed to have a constant value $L_{i}(i=1,2, \ldots, K)$. The circles delimiting these bands are described by equations $\theta=\bar{\theta}_{j}(j=1,2, \ldots$, $K-1$ ), where $\bar{\theta}_{j}$ can be chosen arbitrarily within a set that obeys the inequality

$$
\theta_{i}<\bar{\theta}_{i}<\theta_{i+1}, \quad \bar{\theta}_{0}=0, \quad \bar{\theta}_{K}=\pi .
$$

The zenithal distribution of radiance thus appears as a stepwise constant function:

$$
L(\theta)=L_{i} \quad \text { for } \quad \bar{\theta}_{i-1} \leq \theta<\bar{\theta}_{i} \quad(i=1,2, \ldots K)
$$

(compare Fig. 1). The problem is to calculate all $L_{i}$ on the basis of the measured quantities $M_{i}(i=1$, $2, \ldots, K)$ and known function $h(v)$.

For an individual measurement under zenith angle $\theta=\theta_{i} \leq \pi / 2$, we introduce local Cartesian coordinates $x, y, z$ and also corresponding spherical coordinates $v, \psi$ associated with the point $\mathrm{P}$ and direction $\theta_{i}$, so that $v=\theta-\theta_{i}$ (see Fig. 3). The angular sensitivity of the measurement, $h(v)$, is defined on the whole hemisphere

$$
\sum_{i}:(0 \leq v \leq \pi / 2, \quad 0 \leq \psi \leq 2 \pi)
$$

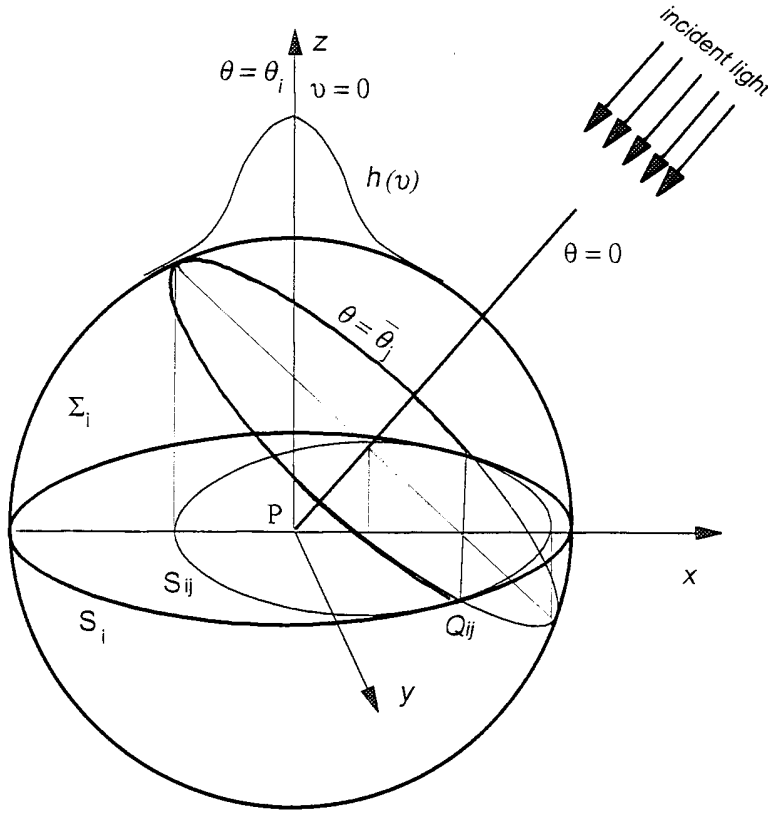

Fig. 3. Geometry of a single measurement in the $\theta=\theta_{i}$ direction. The incident light comes from the $\theta=0$ direction, which is the same as in Fig. 1; however, here the picture is turned clockwise on $\theta_{i} . \quad x, y, z$ is the Cartesian coordinate system associated with this measurement. $\quad \Sigma_{i}$ is the hemisphere of the unit sphere seen from the $\theta=\theta_{i}$ direction. $S_{i}$ is the circumference of radius 1 centered at point $\mathrm{P}$; it is also a projection of the boundary of $\Sigma_{i}$ onto the plane $x P y$. Ellipse $S_{i j}$ is a projection of the delimiting circle $\theta=\bar{\theta}_{j}$ (cf. Fig. 1) onto the plane $x P y . \quad Q_{i j}$ is the point of contact of $S_{i j}$ with $S_{i}$, $v$ is the zenithal spherical coordinate associated with the measurement in the $\theta=\theta_{i}$ direction, and $h(v)$ is the function of the angular sensitivity of the probe.

visible from the direction $\theta_{i}$. As described above, the hemisphere $\Sigma_{i}$ is subdivided into a set of spherical bands $\Sigma_{i j}$ with constant radiance values $L_{j}$. Thus the magnitude $M_{i}$ obtained in this measurement contains contributions from different parts of the hemisphere $\Sigma_{i}$ :

$$
M_{i}=\iint_{\Sigma_{i}} L(\theta) h(v) \mathrm{d} \Sigma=\sum_{j=1}^{n_{i}} L_{j} \iint_{\Sigma_{i j}} h(v) \mathrm{d} \Sigma=\sum_{j=1}^{n_{i}} L_{i} J_{i j}
$$

with

$$
J_{i j}=\iint_{\Sigma_{i j}} h(v) d \Sigma,
$$

where $n_{i}$ is the number of spherical bands contributing to the hemisphere $\Sigma_{i}$.

The system of linear equations, Eq. (3), contains $K$ equations with $K$ unknown variables $L_{j}$. Its coefficients $J_{i j}$ can be estimated because $h(v)$ is a known function. Thus the system (3) provides a solution to our problem. However, the estimation of $J_{i j}$ requires a major effort because the domains of integrations in Eq. (4) are complicated functions of all $\theta_{i}$ and $\bar{\theta}_{j}$, i.e., of the entire geometry of measurements. In 


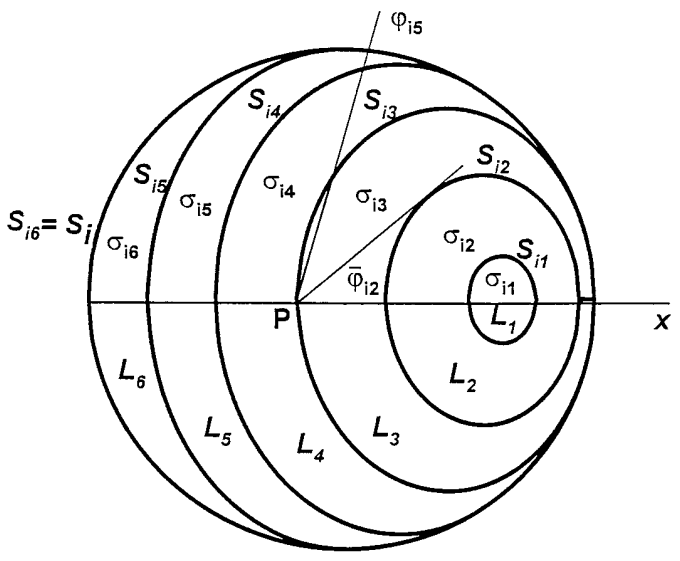

Fig. 4. Mapping of the spherical surface $\Sigma_{i}$ onto the plane $x P y$ performed for the measurement in the $\theta=\theta_{i}$ direction. Circumference $S_{i}$ is the projection of the boundary of the hemisphere $\Sigma_{i}$ onto the plane $x P y$. Ellipses $S_{i j}$ are the projections of circles $\theta=$ $\bar{\theta}_{j}$ onto plane $x P y$. The plane areas $\sigma_{i j}$ are projections of spherical bands $\Sigma_{i j}$ onto plane $x P y$; the index $i$ means only that the mapping is performed within the geometry induced by the $i$ th measurement. $L_{j}$ is the radiance within the band $\Sigma_{i j} . \quad \bar{\varphi}_{i 2}$ is the polar coordinate of the point of tangency for a tangent drawn from point $\mathrm{P}$ to ellipse $S_{i 2} ; \varphi_{i 5}$ is the polar coordinate of the point of contact of ellipse $S_{i 5}$ with the circumference $S_{i}$.

the Subsection 2.B we analyze this geometry in order to facilitate the solution of the integrals $J_{i j}$.

B. Geometry of the Surface Integrals $J_{i j}$

Our starting point is the general relation between the integral of a function $f$ over an arbitrary convex surface $\Sigma$ and that over the plane $\sigma$ :

$$
\iint_{\Sigma} f(M) \mathrm{d} \Sigma=\iint_{\sigma} \frac{f(N)}{\cos \left(n, n_{0}\right)} \mathrm{d} \sigma
$$

where $\left(n, n_{0}\right)$ is the angle between the normal to $\Sigma$ in a point $\mathrm{M}$ and the normal to the plane $\sigma, N$ is the projection of point $\mathrm{M}$ onto the plane, and $f(M)=f(N)$.

To calculate the integrals in Eq. (4) we map the spherical surface $\Sigma_{i}$ onto the plane $x P y$ (see Fig. 3). In this case we have $\left(n, n_{0}\right)=v$, the zenithal spherical coordinate of the local coordinate system associated with the $i$ th measurement; $\Sigma=\Sigma_{i}$, the visible hemisphere corresponding to the $i$ th measurement; and $\sigma$ $=\sigma_{i}$, the circular disk bounded by the circumfernce $S_{i}$ of radius 1 centered at point $\mathrm{P}$.

The mapping transfers the delimiting circles $\theta=\bar{\theta}_{j}$ into ellipses $S_{i j}$ and the surface areas $\Sigma_{i j}$ into the plane areas $\sigma_{i j}$ (compare Figs. 3 and 4 ). On the basis of formulas (4) and (5) we obtain

$$
J_{i j}=\iint_{\sigma_{i j}} \frac{h(v)}{\cos (v)} \mathrm{d} \sigma .
$$

Let us consider projections of the areas $\Sigma_{i j}$ and their boundaries $\theta=\bar{\theta}_{j}$ onto the plane $x P y$. We are not interested in the circles $\theta=\bar{\theta}_{j}\left(\bar{\theta}_{j} \leq \theta_{i}+\pi / 2\right)$ located completely outside the hemisphere $\Sigma_{i}$ and

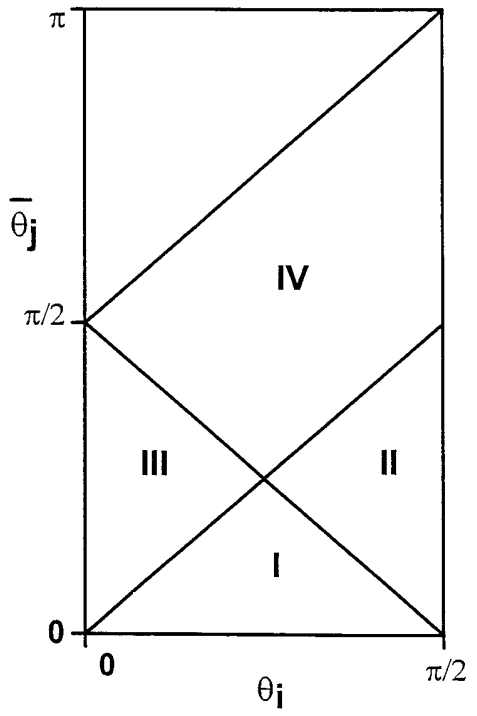

Fig. 5. Admissible domain of values of $\left(\theta_{i}, \bar{\theta}_{j}\right)$, subdivided into four subdomains with a specific relationship between $\theta_{i}$ and $\bar{\theta}_{j}$ in each one.

consider only circles that belong to $\Sigma_{i}$ either completely or partially. In the first case the corresponding ellipses $S_{i j}$ are located completely inside the circular disk $\sigma_{i}$. In the second case they contact the circumference $S_{i}$ in the points $Q_{i j}$ (cf. Figs. 3 and 4 ), and we are interested only in the parts corresponding to those parts of the circles $\theta=\bar{\theta}_{j}$ which are located on the hemisphere $\Sigma_{i}$. We can also restrict the following consideration to the values $\theta_{i} \leq \pi / 2$ because the case $\pi / 2<\theta_{i} \leq \pi$ is transferred to the case $\theta_{i} \leq \pi / 2$ by the coordinate transformation $\theta^{\prime}=\pi-\theta$. Thus the admissible domain of values of $\left(\theta_{i}, \bar{\theta}_{j}\right)$ under consideration is $\left(\theta_{i}<\pi / 2 ; \bar{\theta}_{j}<\theta_{i}+\pi / 2\right)$, which is illustrated in Fig. 5. Also, the sum limit $n_{i}$ in formula (3) can be easily estimated: it is equal to the maximal value of $j$, satisfying the inequality (see Fig. 3)

$$
\bar{\theta}_{j} \geq \theta_{i}+\pi / 2 .
$$

It is more convenient to consider within the circular disk $\sigma_{i}$ a set of nested domains $\bar{\sigma}_{i j}$,

$$
\bar{\sigma}_{i 1} \subset \bar{\sigma}_{i 2} \subset \cdots \subset \bar{\sigma}_{i n_{i}}=\sigma_{i},
$$

instead of the set of nonoverlapping domains $\sigma_{i j}$. Each domain $\bar{\sigma}_{i j}$ is the internal area of the ellipse $S_{i j}$ if this ellipse has no points of contact to $S_{i}$. Otherwise the domain $\bar{\sigma}_{i j}$ is circumferenced by the part of the ellipse $S_{i j}$ contained between its points of contact $Q_{i j}, Q_{i j}{ }^{\prime}$ to the circumference $S_{i}$ and that part of $S_{i}$ located between $Q_{i j}$ and $Q_{i j}{ }^{\prime}$ that is convex toward the positive direction of the axis $x$ (see Fig. 4).

Let us introduce the integrals over the nested domains:

$$
\bar{J}_{i j}=\iint_{\sigma_{i j}} \frac{h(v)}{\cos (v)} \mathrm{d} \sigma .
$$




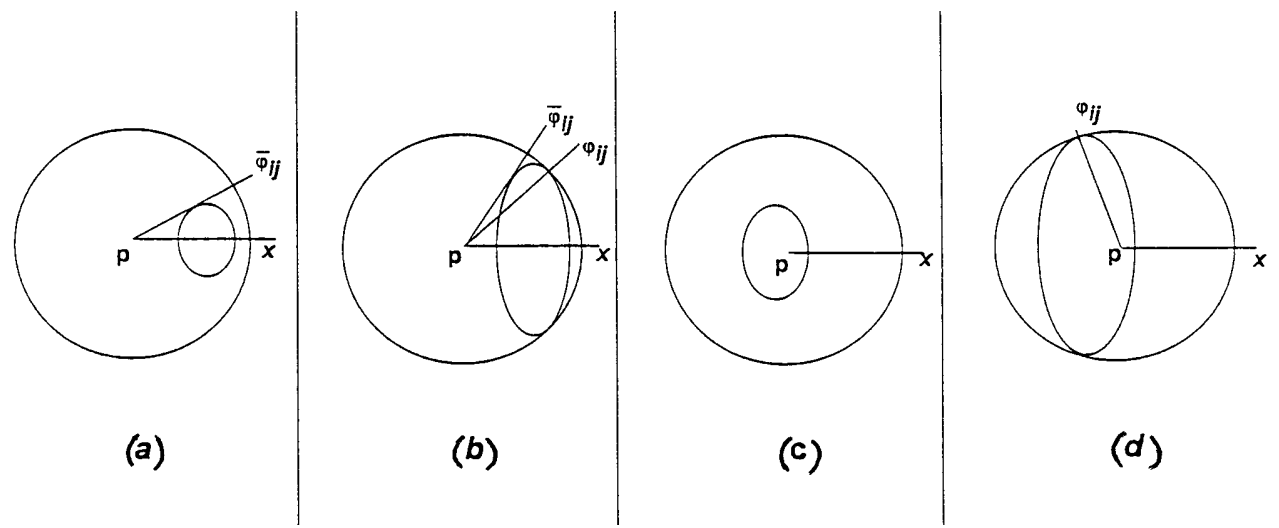

Fig. 6. Different positions of an ellipse $S_{i j}$ with respect to the point $\mathrm{P}$ and circumference $S_{i}$ on plane $x P y$ as implied by the parameter $\theta_{i}, \bar{\theta}_{j}$ representing the geometry of measurements. (a)-(d) correspond to $\theta_{i}, \bar{\theta}_{j}$ values from domains I-IV in Fig. 5.

Obviously

$$
J_{i j}=\bar{J}_{i j}-\bar{J}_{i j-1}\left(j=1,2, \ldots, n_{i}\right),
$$

where (cf. Fig. 4)

$$
\bar{J}_{i 0}=0, \quad \bar{J}_{i n_{i}}=2 \pi \int_{0}^{1} \frac{h(v)}{\cos v} r \mathrm{~d} r .
$$

Thus the integrals $J_{i j}$ can be easily calculated from integrals $\bar{J}_{i j}$ that have much more convenient integration domains. However, prior to this the limits of the surface integrals should be analytically derived. This derivation and the subsequent calculation of $\bar{J}_{i j}$ are given in Subsection 2.C.

\section{Calculation of Integrals $\bar{J}_{i j}$}

For the integration limits to be derived, the boundaries of the domains $\bar{\sigma}_{i j}$ should be described. We introduce polar coordinates $r, \varphi$ (note that $r=\sin v$ ) on the plane $x P y$ and also the double-valued function $f_{i j}^{-}(\varphi)$ and $r=f_{i j}^{+}(\varphi)$. In this case the point of tangency is located on the boundary of a domain $\bar{\sigma}_{i j}$ and

$$
\cos \bar{\varphi}_{i j}=\frac{\left[\sin \left(\theta_{i}+\theta_{j}\right) \sin \left(\theta_{i}-\theta_{j}\right)\right]^{1 / 2}}{\sin \theta_{i}} .
$$

If $\bar{\theta}_{j}>\theta_{i}$ then the ellipse $S_{i j}$ is located within the negative semiaxis $x$. In this case only the branch $r=$ $f_{i j}^{+}(\varphi)$ makes a part of the boundary of $\bar{\sigma}_{i j}$ and the point of tangency is not located on the boundary.

The important boundary point for integration to be performed is the point of contact for an ellipse $S_{i j}$ with the circumference $S_{i j}$. If such a point exists it is always located, as one can easily see, on a positive branch $r=f_{i j}{ }^{+}(\varphi)$. The $\varphi$ coordinate of this point we designate as $\varphi_{i j}$. The value of $\varphi_{i j}$ is determined through the parameters $\theta_{i}, \bar{\theta}_{j}$ as (see Appen$\operatorname{dix}$ A)

$$
\cos \varphi_{i j}=\cos \bar{\theta}_{j} / \sin \theta_{i}
$$

$$
f_{i j}^{ \pm}(\varphi)=\frac{\cos \bar{\theta}_{j} \sin \theta_{i} \cos \varphi \pm \cos \theta_{i}\left[\sin \left(\theta_{i}+\theta_{j}\right) \sin \left(\theta_{j}-\theta_{i}\right)+\sin ^{2} \theta_{i} \cos ^{2} \varphi\right]^{1 / 2}}{\cos ^{2} \theta_{i}+\sin ^{2} \theta_{i} \cos ^{2} \varphi}
$$

which will be useful for the description of the ellipses $S_{i j}$.

An ellipse $S_{i j}$ contains the coordinates origin $\mathrm{P}$ when the parameters $\theta_{i}, \bar{\theta}_{j}$ satisfy the inequality

$$
\sin \left(\theta_{i}+\bar{\theta}_{j}\right) \sin \left(\theta_{i}-\bar{\theta}_{j}\right)<0,
$$

i.e., $\theta_{i}<\bar{\theta}_{j}<\pi-\theta_{i}$. In this case its equation is presented by the single-valued function $r=f_{i j}{ }^{+}(\varphi)$ (for the proof see Appendix A). Otherwise point $\mathrm{P}$ is located outside the ellipses $S_{i j}$. In such a case a tangent line from point $\mathrm{P}$ to the ellipse is always feasible. We designate the $\varphi$ coordinate of the point of tangency as $\bar{\varphi}_{i j}$ (cf. Fig. 4).

If the ellipse is located completely within the positive semiaxis $x$ (which takes place for $\bar{\theta}_{j}<\theta_{i}$ ), then to any value $0<\varphi<\bar{\varphi}_{i j}$ correspond two values of $r: r=$
On the basis of the above considerations, four different cases appear with respect to the subdivision of the integration domain for an integral $\bar{J}_{i j}$ and elucidation of the integration limits in each subdomain. Accordingly, the admissible domain of parameters $\theta_{i}, \bar{\theta}_{j}$ is subdivided into four subdomains, shown in Fig. 5. Below we consider all four cases and derive explicite formulas for $\bar{J}_{i j}$ in each case.

Case I. $\bar{\theta}_{j}<\theta_{i}, \bar{\theta}_{j} \leq \pi / 2-\theta_{i}$. Ellipse $S_{i j}$ is located completely on the positive semiaxis $x$ and has no points of contact with $S_{i}$ (or the unique point of contact has coordinate $\varphi_{i j}=0$ ). This case is depicted in Fig. 6(a).

$$
\bar{J}_{i j}=2 \int_{0}^{\bar{\varphi}_{i j}} \mathrm{~d} \varphi \int_{f_{i j}^{+}(\varphi)}^{f_{i j}-(\varphi)} \frac{h(v)}{\cos (v)} r d r .
$$


Case II. $\quad \bar{\theta}_{j}<\theta_{i}, \bar{\theta}_{j}>\pi / 2-\theta_{i}$. The ellipse $S_{i j}$ is located on the positive semiaxis $x$ and has the point of contact $\varphi_{i j} \neq 0$ with $S_{i}$ [see Fig. $6(\mathrm{~b})$ ].

$$
\begin{aligned}
\bar{J}_{i j}= & 2 \int_{0}^{\varphi_{i j}} \mathrm{~d} \varphi \int_{f_{i j}-(\varphi)}^{1} \frac{h(v)}{\cos (v)} r \mathrm{~d} r \\
& +2 \int_{\varphi_{i j}}^{\bar{\varphi}_{i j}} \mathrm{~d} \varphi \int_{f_{i j}-(\varphi)}^{f_{i j^{+}}(\varphi)} \frac{h(v)}{\cos (v)} r \mathrm{~d} r .
\end{aligned}
$$

Case III. $\quad \bar{\theta}_{j}>\theta_{i}, \bar{\theta}_{j} \leq \pi / 2-\theta_{i} . \quad$ Point $\mathrm{P}$ is located inside the ellipse $S_{i j}$, which has no point of contact with $S_{i}$ [or the unique point of contact has coordinate $\varphi_{i j}=0$; see Fig. 6(c)].

$$
\bar{J}_{i j}=2 \int_{0}^{\pi} \mathrm{d} \varphi \int_{0}^{f_{i j}+(\varphi)} \frac{h(v)}{\cos (v)} r \mathrm{~d} r .
$$

Case IV. $\bar{\theta}_{j}>\theta_{i}, \bar{\theta}_{j}>\pi / 2-\theta_{i}$. Ellipse $S_{i j}$ is located completely or partially on the negative semiaxis $x$ and has points of contact $\pm \varphi_{i j} \neq 0$ [see Fig. $6(d)]$.

$$
\bar{J}_{i j}=2 \int_{0}^{\pi} \mathrm{d} \varphi \int_{0}^{1} \frac{h(v)}{\cos (v)} r \mathrm{~d} r-2 \int_{\varphi_{i j}}^{\pi} \mathrm{d} \varphi \int_{f_{i j}+(v)}^{1} \frac{h(v)}{\cos (v)} r \mathrm{~d} r .
$$

Substituting Eq. (2) for $h(v)$ into Eqs. (12) and noting that $r=\sin v$, we can easily obtain a general solution for the internal integral in formulas (12):

$$
\begin{aligned}
\int_{f_{1}(\varphi)}^{f_{2}(\varphi)} \exp \left(-m r^{2}\right) r \mathrm{~d} r= & \frac{1}{2 m}\left\{\exp \left[-m f_{1}^{2}(\varphi)\right]\right. \\
& \left.-\exp \left[-m f_{2}^{2}(\varphi)\right]\right\} .
\end{aligned}
$$

Thus for any set of $\theta_{i}, \bar{\theta}_{j}$ presenting a concrete geometry of measurements and for a given $m$ presenting the directional sensitivity of the concrete microprobe, integrals (12) and then [using Eq. (8)] integrals (4) can be calculated. Substituting integrals (4) into Eq. (3) and solving these equations with respect to $L_{i}(i=1,2, \ldots, K)$, one obtains the real radiances from the measured quantites $M_{i}$.

Note that when the measured radiation field contains a strong collimated component, for example, under illumination with a laser, the calculation of integrals $J_{i 1}$ can be developed in a special way involving the description of the collimated component as a singularity by means of $\delta$ functions. This appears to be necessary when the measurements are planed and discussed in the framework of the theory of radiative transfer, in which the collimated component is aways separated as a singularity. The appropriate procedure for the calculation of $J_{i 1}$ is given in Appendix B.

\section{Example of Application: Correction of the} Measurements and Stability of the Correcting Procedure

Here the theory is applied to our radiance measurements in costal sediments with diatoms. Proceeding

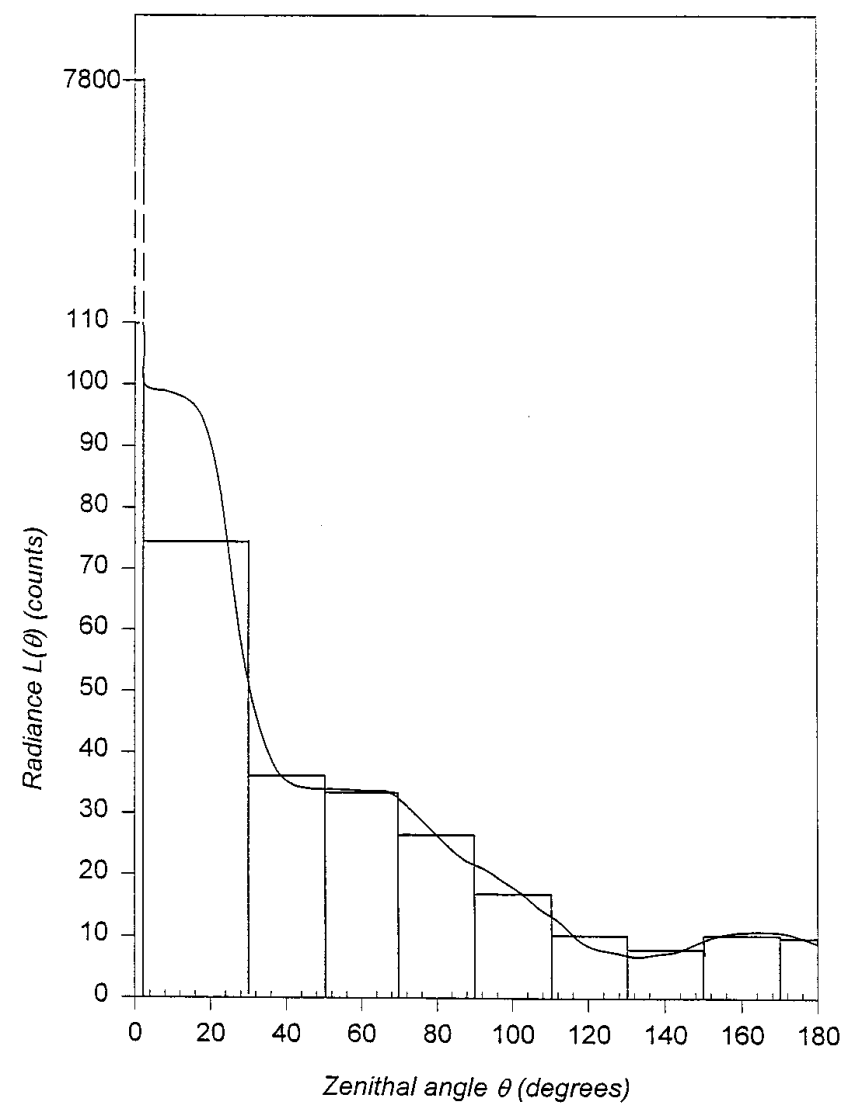

Fig. 7. True radiance distribution $L(\theta)$. This distribution with the fluence rate value of $I(P)=349.9$ relative units is the starting point for the analysis of the correcting procedure. Averaged values $\bar{L}_{i}$ for spherical bands (specified by vertical lines) calculated on the basis of this distribution are used in Table 1.

from a true radiance distribution, solid $L(\theta)$, and corresponding fluence rate $I(\mathrm{P})=\int L(\theta) \mathrm{d} \theta$, we find estimates of $L(\theta)$ and $I(P)$ (a) as the raw experimental data and (b) as the data processed by the theory, and then we compare estimates (a) and (b) one to another and to the true magnitudes. Furthermore, we repeat this procedure for probes with four different acceptance angles $\theta_{a}$ as well as for different angular distances between the measurements in order to reveal the effect of these characteristics on both processed and nonprocessed data.

We start with the true radiance distribution $L(\theta)$ shown in Fig. 7. Its diffuse part is a continuous function; the collimated part we interpret as a constant level radiance concentrated in the narrow angular range $\left(0,2.5^{\circ}\right)$. The fluence rate of this radiation $I(\theta)=349.9$ relative units. ${ }^{12}$ On the basis of known $L(\theta)$, the measurable quantity $M(\theta)$ can be calculated for any direction $\theta_{i}$ and any microprobe as

$$
\iint_{\Sigma_{i}} L(\theta) h\left(\theta-\theta_{i}\right) \mathrm{d} \Sigma=M_{i}
$$

This quantity calculated for four different $m$ values is shown in Fig. 8. Because of a very extended range of values of $M(\theta)$, the angular domain near $\theta=0^{\circ}$ is 


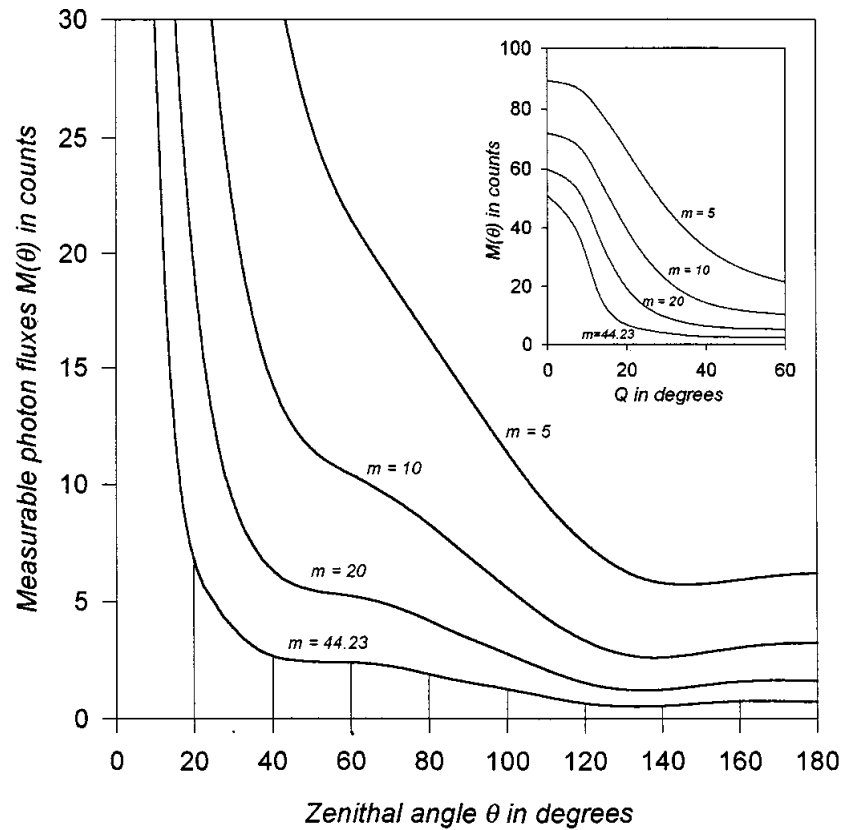

Fig. 8. Zenithal distributions of the measurable photon fluxes $M(\theta)$ based on the true radiance distribution $L(\theta)$ as they can be obtained by four microprobes with a different parameter $m$. Specified points of $\theta_{i}=0^{\circ}, 20^{\circ}, 40^{\circ}, \ldots, 180^{\circ}$ were used to perform the correction procedure. The initial part of the curves $M(\theta)$ for the lower $\theta$ range is shown in the inset.

shown separately in the inset at a different scale. On each curve in Fig. 8 one can read the fluxes (ordinate) measured by the corresponding microprobe oriented in the direction $\theta$ (abscissa) when the true distribution solid of radiance is presented by the function $L(\theta)$ from Fig. 7. Of course, the real measurements are performed only on a restricted number of points, providing an approximative shape of the curves in Fig. 8. For example, the measurements spaced equidistantly with the $20^{\circ}$ step and performed with the probe having $m=44.23$ are specified in Fig. 8. A comparison of the curves in Figs. 7 and 8 shows that the nonprocessed measurements give a severely distorted picture of the radiance distribution solid, especially those with larger acceptance angles of the probes. Only the probe with the most peaked directional sensitivity (Fig. 8, lowest curve) conveys more or less the shape of $L(\theta)$, although this is rather distorted and has strongly underestimated values.

Now let us process these data according to the theory. We consider the set of equidistant measurements with the $20^{\circ}$ step in ten directions $\theta_{i}\left(0^{\circ}\right.$, $20^{\circ}, \ldots, 180^{\circ}$ ) for all four probes, represented in Fig. 8. We subdivide the unit sphere into ten spherical bands with assumed constant radiance value $L_{i}$ within each band. The numbers and zenithal areas of these bands are given in columns 1 and 2 of Table 1 . Note that the first band is very narrow because we assume that the light within this zenithal area contains the nonscattered part of the incident collimated flux and can strongly differ from the surroundings. Then we perform the entire correcting procedure to
Table 1. Radiance Values $L_{i}$ Recovered by Means of the Correcting Procedure Applied to the Measured Fluxes $M_{i}$ from the Curves in Fig. 8

\begin{tabular}{rcrrrrr}
\hline & \multirow{2}{*}{$\begin{array}{c}\text { Band } \\
\text { Band }\end{array}$} & $\begin{array}{c}\text { Range } \\
\text { No. }\end{array}$ & & & \multicolumn{5}{c}{$L_{i}$ Values } \\
\cline { 5 - 8 } & $($ deg$)$ & $\bar{L}_{i}$ & $m=44.23$ & $m=20$ & $m=10$ & $m=5$ \\
\hline 1 & $0-2.5$ & 7800 & 8034 & 8229 & 8371 & 8186 \\
2 & $2.5-30$ & 74.5 & 78.1 & 77.7 & 76.7 & 80.7 \\
3 & $30-50$ & 36.1 & 34.3 & 32.8 & 31.9 & 26.6 \\
4 & $50-70$ & 33.5 & 33.9 & 34.5 & 35.6 & 42.0 \\
5 & $70-90$ & 25.4 & 25.4 & 25.5 & 25.0 & 29.0 \\
6 & $90-110$ & 16.8 & 16.7 & 16.6 & 16.8 & 20.7 \\
7 & $110-130$ & 8.5 & 8.0 & 7.8 & 7.8 & 4.6 \\
8 & $130-150$ & 8.0 & 7.8 & 7.7 & 7.7 & 10.0 \\
9 & $150-170$ & 10.7 & 10.9 & 11.1 & 11.2 & 9.1 \\
10 & $170-180$ & 9.6 & 9.7 & 9.5 & 9.0 & 14.8 \\
\hline
\end{tabular}

${ }^{a}$ For each of four curves representing different microprobes, the $M_{i}$ values corresponding to the ten equidistant points $\theta_{i}$ with the $20^{\circ}$ step $\left(0^{\circ}, 20^{\circ} \ldots, 180^{\circ}\right)$ were used. $\bar{L}_{i}$ is the true mean value as shown in Fig. 7.

find the $L_{i}$ from the $M_{i}$. The results for the four probes are given in columns 4 to 7 of Table 1 . These results should be compared with the true radiance values $L(\theta)$ averaged over each band, presented in column 3 of Table 1 as $\bar{L}_{i}$. Figure 7 shows the averaged true values $\bar{L}_{i}$ on the background of the radiance distribution $L(\theta)$. As one can see from Table 1 , the correcting procedure recovers the radiance distribution solid with extremely high accuracy. The relative deviation of $L_{i}$ from $\bar{L}_{i}$ is in almost all cases within $\pm 5 \%$.

Let us address the estimates of the fluence rate $I(P)$ that are presented in Table 2 . Column 1 contains the parameters of the four probes under consideration. Columns 2-4 contain the characteristics of the equidistant measurements constructed in accordance with each probe. For example, the probe with $\theta_{a}=7.2^{\circ}$ implies the step $\Delta=15^{\circ}$ between measurements. Correspondingly, the number of measurements is $K=2 \pi / \Delta+1=13$. The reference area of a microprobe (see Section 1) for this band size is $S=$ 0.0538. Note that the rather artificial magnitude $S$ is necessary only for nonprocessed calculations to proceed from the measured flux $M_{i}$ to the radiance $L_{i}$. In the framework of the theory, there is no place for the notion of $S$ because the reference area is the entire hemisphere $\Sigma_{i}$ and the transmission from the measured fluxes $M_{i}$ to the radiance $L_{i}$ is accomplished automatically through the integration and solution of system (3). Column 5 contains the results provided by the measurements characterized in columns 2-4. All these numbers strongly overestimate the true value $I(P)=349.9$ presented above. In order to see the effect of deviations of the band size from the probe acceptance angle, we performed calculations with the fixed band size $\left(20^{\circ}\right)$ and corresponding number of measurements (ten) for different probes (column 6). The probe with $\theta_{\alpha}=7.2^{\circ}$ used under these conditions underestimates the fluence rate; the probes with $\theta_{a}=15.1^{\circ}$ and $\theta_{a}=21.2^{\circ}$ that have acceptance angles exceeding the band size 
Table 2. Processed and Nonprocessed Fluence Rate Values $I(\boldsymbol{P})^{a}$

\begin{tabular}{|c|c|c|c|c|c|c|}
\hline \multirow{3}{*}{$\begin{array}{c}\text { Probe } \\
\text { Parameters }\end{array}$} & \multicolumn{5}{|c|}{ Nonprocessed Results } & \multirow{3}{*}{ 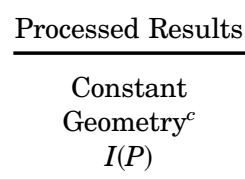 } \\
\hline & \multicolumn{4}{|c|}{ Equidistant Measurements ${ }^{b}$} & \multirow{2}{*}{$\begin{array}{c}\text { Constant } \\
\text { Geometry }^{c} \\
(S=0.0954) I(P)\end{array}$} & \\
\hline & $S$ & $\Delta$ & $K$ & $I(P)$ & & \\
\hline $\begin{aligned} m & =44.23 \\
\theta_{a} & =7.2^{\circ}\end{aligned}$ & 0.0538 & $15^{\circ}$ & 13 & 466.9 & 274.2 & 351.0 \\
\hline $\begin{aligned} m & =20 \\
\theta_{a} & =10.7^{\circ}\end{aligned}$ & 0.0954 & $20^{\circ}$ & 10 & 581.8 & 581.8 & 350.6 \\
\hline $\begin{aligned} m & =10 \\
\theta_{a} & =15.1^{\circ}\end{aligned}$ & 0.2141 & $30^{\circ}$ & 7 & 520.8 & 1165.0 & 350.5 \\
\hline $\begin{aligned} m & =5 \\
\theta_{a} & =21.2^{\circ}\end{aligned}$ & 0.4783 & $45^{\circ}$ & 5 & 462.2 & 2308.2 & 350.9 \\
\hline
\end{tabular}

${ }^{a}$ These values are obtained from the same sets of measured fluxes $M_{i}$ as used in Table $1 . \quad S$ is the reference area of the probe; $\Delta \approx 2 \theta_{a}$ is the step between measurements; $K$ is the number of measurements. The true value of $I(P)$ is 349.9 relative units.

${ }^{b}$ Geometry is adjusted to the band size.

${ }^{c}$ Here $\Delta=20^{\circ}$ and $K=10$.

highly overestimate it. Column 7 of Table 2 describes the same measurements as column 6, with the difference that the data were processed by the theory. The estimates in column 7 are very precise, with deviations from the true value within $1 \%$, independent of whether the band size is adjusted to the acceptance angle of the probe (line 2) or not (lines 1 , 3 , and 4).

\section{Appendix A: Geometry of Ellipses $S_{i j}$}

We consider the measurement $\theta_{i}$ and the corresponding circular disk $S_{i}$ normal to the direction $\theta=\theta_{i}$. The circumference $\theta=\bar{\theta}_{j}$ on the unit sphere is mapped onto the plane $S_{i}$ as the ellipse $S_{i j}$. Let us designate the half-axes of $S_{i j}$ as $a_{i j}, b_{i j}$ and the $x$ coordinate of the center as $q_{i j}$ (see Fig. 9). As we can easily see from Fig. 9,

$$
\begin{aligned}
a_{i j} & =\sin \bar{\theta}_{j} \cos \theta_{i}, \\
b_{i j} & =\sin \bar{\theta}_{j}, \\
q_{i j} & =\cos \bar{\theta}_{j} \sin \theta_{i}, \\
c_{i j}{ }^{2} & =b_{i j}{ }^{2}-a_{i j}{ }^{2}=\sin ^{2} \bar{\theta}_{j} \sin ^{2} \theta_{i} .
\end{aligned}
$$

The equation of the ellipse $S_{i j}$ in the polar coordinates $(r, \varphi)$ is given by expressions (see the appendix in Ref. 10) $r=f_{i j}{ }^{+}(\varphi)$ when point $\mathrm{P}$ is inside $S_{i j}$ and $r=f_{i j}{ }^{ \pm}(\varphi)$ when point $\mathrm{P}$ is outside $S_{i j}$, where
Point P is located inside the ellipse $S_{i j}$ when $a_{i j}{ }^{2}-$ $q_{i j}{ }^{2}>0$, which yields the condition $\sin \left(\theta_{i}+\bar{\theta}_{j}\right) \sin \left(\theta_{j}-\right.$ $\left.\theta_{i}\right)>0$, i.e., $\pi-\theta_{i}>\bar{\theta}_{j}>\theta_{i}$. Outside the range where this condition holds the inequality $\sin \left(\theta_{i}+\right.$ $\left.\bar{\theta}_{j}\right) \sin \left(\theta_{i}-\bar{\theta}_{j}\right)>0$ is valid.

Let us consider the area $\theta_{i}>\bar{\theta}_{j}$, i.e., the case in which the ellipse $S_{i j}$ is completely on the positive semiaxis $x$. We draw a tangent to the ellipse $S_{i j}$ from point $\mathrm{P}$. The coordinate of the point of tangency $\bar{\varphi}_{i j}$ is found from the condition for vanishing of the radical in Eq. (A3): $\sin \left(\theta_{i}+\bar{\theta}_{j}\right) \sin \left(\bar{\theta}_{j}-\theta_{i}\right)+\sin ^{2} \theta_{i} \cos ^{2} \varphi=0$, which yields

$$
\cos \bar{\varphi}_{i j}=\frac{\left[\sin \left(\theta_{i}+\bar{\theta}_{j}\right) \sin \left(\theta_{i}-\bar{\theta}_{j}\right)\right]^{1 / 2}}{\sin \theta_{i}} .
$$

If the ellipse $S_{i j}$ has a point of contact with disk $S_{i}$, its coordinate $\varphi=\varphi_{i j}$ is found from the condition $f_{i j}{ }^{+}$ $=1$, which holds when $\cos \varphi_{i j}=\cos \bar{\theta}_{j} / \sin \theta_{i}$.

Appendix B: Calculation of integrals $J_{i 1}$

This calculation is of integrals $J_{i 1}$ when the collimated component is presented as a singularity by means of a $\delta$ function. The collimated component of radiance $F_{c}$ can be presented as $F_{c}=F_{p} \delta(\mu-1)$, where

$F_{p}$ is the collimated flux at point $\mathrm{P}$, $\mu=\cos \theta$,

$\int_{0}^{1} \delta(\mu-1) \mathrm{d} \mu=1$,

$$
f_{i j}^{ \pm}=\frac{q_{i j} b_{i j}{ }^{2} \cos (\varphi) \pm a_{i j} b_{i j}\left[a_{i j}{ }^{2}-q_{i j}{ }^{2}+\left(q_{i j}{ }^{2}+c_{i j}{ }^{2}\right) \cos ^{2}(\varphi)\right]^{1 / 2}}{a_{i j}{ }^{2}+c^{2} \cos ^{2}(\varphi)} .
$$

Substituting Eq. (A1) into Eq. (A2), we obtain

$\int_{0}^{1} f(\mu) \delta(\mu-1) \mathrm{d} \mu=f(1)$.

$$
f_{i j}^{ \pm}(\varphi)=\frac{\cos \bar{\theta}_{j} \sin \theta_{i} \cos \varphi \pm \cos \theta_{i}\left[\sin \left(\theta_{i}+\theta_{j}\right) \sin \left(\theta_{j}-\theta_{i}\right)+\sin ^{2} \theta_{i} \cos ^{2} \varphi\right]^{1 / 2}}{\cos ^{2} \theta_{i}+\sin ^{2} \theta_{i} \cos ^{2} \varphi} .
$$




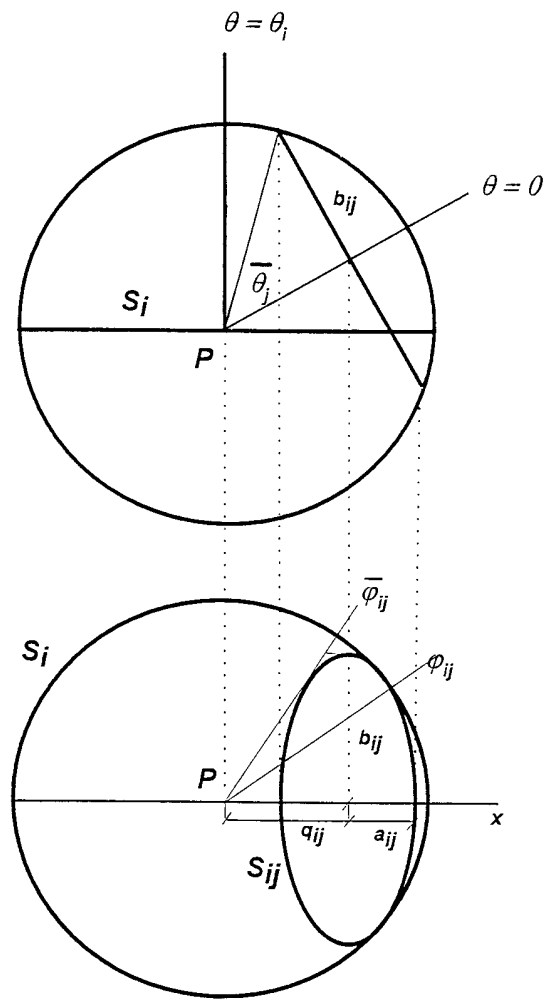

Fig. 9. Derivation of formulas for the half-axes $a_{i j}, b_{i j}$ of the ellipse $S_{i j}$ and the distance $q_{i j}$ between the point $\mathrm{P}$ and the center of $S_{i j}$. All designations are the same as in Figs. 3, 5, and 7. The top section shows the vertical cross section of the unit sphere containing the directions $\theta=0$ and $\theta=\theta_{i}$. The bottom section shows the cross section of the unit sphere through the plane $x P y$ normal to the direction $\theta=\theta_{i}$.

For a measurement in the direction $\theta_{i} \leq 90^{\circ}$, one should specify

$$
\begin{aligned}
& L_{1}=F_{p}, \\
& J_{i 1}=\int_{0}^{1} h(v) \delta(\mu-1) \mathrm{d} \mu .
\end{aligned}
$$

For $h(v)=\cos v \exp \left(-m \sin ^{2} v\right)$ we obtain

$$
\begin{aligned}
& v=\theta-\theta_{i}, \\
& \cos v=\mu \cos \theta_{i}+\sqrt{1-\mu^{2}} \sin \theta_{i}, \\
& \sin v=\sqrt{1-\mu^{2}} \cos \theta_{i}-\mu \sin \theta_{i}, \\
& J_{i 1}=\cos \theta_{i} \exp \left(-m \sin ^{2} \theta_{i}\right) .
\end{aligned}
$$

This research was supported by grant Fu 152/6-2 from Deutsche Forschungs-gemeinschaft, the Max-Planck Gesellschaft, and the Carlsberg foundation, Denmark (for B. Jørgensen). This paper is dedicated to P-S. Song on the occasion of his 60th birthday.

\section{References and Notes}

1. T. C. Vogelmann and L. O. Björn, "Measurements of light gradients and spectral regime in plant tissue with a fiber optic probe," Physiol. Plant. 60, 361-368 (1984).

2. B. B. Jørgensen and D. J. Des Marais, "A simple fiber-optic microprobe for high resolution light measurements: application in marine sediment," Limnol. Oceanogr. 31, 1376-1383 (1986).

3. L. Lilge, T. Haw, and B. C. Wilson, "Miniature isotropic optical fibre probes for quantitative light dosimetry in tissues," Phys. Med. Biol. 38, 215-230 (1987).

4. T. C. Vogelmann, G. Martin, G. Chen, and D. Buttry, "Fibre optic microprobes and measurement of the light microenvironment within plant tissues," Adv. Bot. Res. 18, 255-295 (1991).

5. L. Fukshansky, N. Fukshansky-Kazarinova, and A. Martinez v. Remisowsky, "Estimation of optical parameters in a living tissue by solving the inverse problem of the multi-flux radiative transfer," Appl. Opt. 30, 3145-3153 (1991).

6. M. Kühl and B. B. Jørgensen, "Spectral light measurements in microbenthic communities with a fiber-optic microprobe coupled to a sensitive diode array detector system," Limnol. Oceanogr. 37, 1813-1823 (1992).

7. M. Kühl and B. B. Jørgensen, "The light field of microbenthic communities: radiance distribution and microscale optics of sandy coastal sediments," Limnol. Oceanogr. 39, 1368-1398 (1994).

8. M. Kühl, C. Lassen, and B. B. Jørgensen, "Optical properties of microbial mats: light measurements with fiber-optic microprobes," in Microbial mats: Structure, Development and Environmental Significance, L. J. Stal and P. Gaumette, eds., NATO ASI Series G (Springer-Verlag, Berlin, 1994), pp. 149166.

9. T. Richter and L. Fukshansky, "Optics of bifacial leaf: 1. A novel procedure for derivation of the optical parameters," J. Photochem. Photobiol. 63, 507-516 (1996).

10. N. Fukshansky-Kazarinova, L. Fukshansky, M. Kühl, and B. B. Jørgensen, "Theory of equidistant three-dimensional radiance measurements with optical microprobes," Appl. Opt. 35, 65-73 (1995).

11. J. M. Senior, Optical Fiber Communications: Principles and Practice (Prentice-Hall, Englewood Cliffs, N.J., 1985).

12. By calibrating the microprobe against a standard source, we can express the measured values in absolute units of $\mathrm{W} \mathrm{m} \mathrm{m}^{-2} \mathrm{~s}^{-1} \mathrm{sr}^{-1}$ for $L_{i}$ and $\mathrm{W} \mathrm{m}^{-2} \mathrm{~s}^{-1}$ for $I(P)$. However, this is not necessary for our purposes. 